\title{
Quasi-Exact Solvability of a Hyperbolic Intermolecular Potential Induced by an Effective Mass Step
}

\author{
Axel Schulze-Halberg \\ Department of Mathematics and Actuarial Science, Indiana University Northwest, 3400 Broadway, \\ Gary, IN 46408, USA \\ Correspondence should be addressed to Axel Schulze-Halberg, xbataxel@gmail.com
}

Received 3 December 2010; Accepted 11 January 2011

Academic Editor: N. Govil

Copyright (C) 2011 Axel Schulze-Halberg. This is an open access article distributed under the Creative Commons Attribution License, which permits unrestricted use, distribution, and reproduction in any medium, provided the original work is properly cited.

It is shown that a nonsolvable third-order hyperbolic potential becomes quasi-exactly solvable after the introduction of a hyperbolic effective mass step. Stationary energies and $L^{2}$-solutions of the corresponding Schrödinger equation are obtained in explicit form.

\section{Introduction}

Hyperbolic potentials are commonly used to model interatomic and intermolecular forces. Most famous examples of such potentials include the Pöschl-Teller, the Rosen-Morse, and the Scarf-type potentials, which have been studied extensively. Many of these hyperbolic potentials are exactly solvable or quasi-exactly solvable; the corresponding closed-form Schrödinger eigenfunctions and stationary energies can be found, for example, in [1$3]$ and the references therein. In this paper, we study solvability hyperbolic potentials in combination with effective (position-dependent) masses. Effective masses occur in the context of transport phenomena in crystals (e.g., semiconductors), where the electrons are not completely free, but interact with the potential of the lattice. The quantum dynamics of such electrons can be modeled by an effective mass, the behaviour of which is determined by the band curvature [4-6]. The solvability of the Schrödinger equations with effective mass and hyperbolic potentials has been studied recently using point canonical transformations [7-9] and supersymmetry-based factorization [10,11]. In comparison with the constant mass case, the effective-mass Schrödinger equations are less likely to be solvable for potentials of physical interest. This is so because the effective mass function-which must be chosen to 
be physically meaningful—gives the equation a more complicated form than in the constantmass case. Thus, the solvable Schrödinger equation does not in practice remain solvable after the introduction of an effective mass. The purpose of this paper is to observe the opposite situation. We consider the Schrödinger equation for a nonsolvable, hyperbolic potential of order three that generalizes many well-known potentials, including the Pöschl-Teller and the Rosen-Morse potential. We combine our potential with an effective mass step that has proved useful in studying the effective-mass Schrödinger equations [7, 12, 13]. After the introduction of the effective mass, the corresponding Schrödinger equation becomes quasi-exactly solvable and even admits $L^{2}$-solutions. In Section 2 , we summarize our results, stating the stationary energies and the corresponding solutions of the effective mass Schrödinger equation. We also give conditions for the solutions to be $L^{2}$-normalizable. In Section 3 , the results are proved.

\section{Statement of Results}

The stationary Schrödinger equation with effective mass $m$ and potential $V$ has the following general form [14]:

$$
\frac{1}{2 m(x)} \Psi^{\prime \prime}(x)-\frac{m^{\prime}(x)}{2 m^{2}(x)} \Psi^{\prime}(x)+(E-V(x)) \Psi(x)=0
$$

We take the effective mass to be a smooth step function:

$$
m(x)=1+\tanh (x)
$$

which, as mentioned in the Introduction, has been used frequently in studies of effective mass problems. Now, we define the potential in (2.1) as follows:

$$
V_{a, b, c, d}(x)=a \tanh (x)+b \tanh ^{2}(x)+c \tanh ^{3}(x)+d,
$$

where $a, b, c$, and $d$ are real numbers. Potential (2.3) and its special cases are common models for intermolecular forces; as typical examples let us mention the following subcases that have been studied before regarding their solvability $[1,2]$ :

$$
\begin{gathered}
V_{a, b, 0, a+b}(x)=a(1+\tanh (x))+b\left(1+\tanh (x)^{2}\right), \\
V_{a, b, 0,-b}(x)=a \tanh (x)-b \frac{1}{\cosh ^{2}(x)}, \\
V_{0, a, 0,0}(x)=a \tanh ^{2}(x), \\
V_{0,-a, 0, a}(x)=a \frac{1}{\cosh ^{2}(x)} .
\end{gathered}
$$

Potentials (2.5) and (2.7) are the Rosen-Morse potential and the Pöschl-Teller potential, respectively. It is well known that the Schrödinger equation (2.1) for constant mass and 
potential (2.3) is not solvable. However, it becomes solvable if we introduce the effective mass (2.2).

\subsection{The Stationary Energies}

Throughout this paper we assume that the energy $E$ in the Schrödinger equation (2.1) satisfies the following relation:

$$
E=b-a+d-c
$$

where $a, b, c$, and $d$ are the coupling constants of potential (2.3). Since the latter potential depends on the value of the energy (2.8), the corresponding Schrödinger equation can be at most quasi-exactly solvable. As we will see below, for a given setting of the coupling constants $a, b, c, d$, there is exactly one solution available.

\subsection{Solution and $L^{2}$-Normalizability for $c \neq 0$}

Under condition (2.8) and $c \neq 0$, the Schrödinger equation (2.1) with effective mass (2.2) and potential (2.3) admits the following complete solution:

$$
\Psi(x)=C_{1} \Psi_{1}(x)+C_{2} \Psi_{2}(x),
$$

where $C_{1}, C_{2}$ are constants and the functions $\Psi_{1}, \Psi_{2}$ are given by

$$
\begin{aligned}
\Psi_{1}(x)= & \exp (\sqrt{2 c}(\tanh (x)-1))(\tanh (x)-1)^{\sqrt{2(a+c)}} \\
& \times U\left(-\frac{b+c}{\sqrt{2 c}}+\sqrt{2(a+c)}+\frac{1}{2}, \sqrt{8(a+c)}+1, \sqrt{8 c}(1-\tanh (x))\right), \\
\Psi_{2}(x)= & \exp (\sqrt{2 c}(\tanh (x)-1))(\tanh (x)-1)^{\sqrt{2(a+c)}} \\
& \times{ }_{1} F_{1}\left(-\frac{b+c}{\sqrt{2 c}}+\sqrt{2(a+c)}+\frac{1}{2}, \sqrt{8(a+c)}+1, \sqrt{8 c}(1-\tanh (x))\right) .
\end{aligned}
$$

Here, $U$ and ${ }_{1} F_{1}$ denote the usual confluent hypergeometric functions [15]. We have the following conditions for $L^{2}$-normalizability.

(i) The solution $\Psi_{1}$ given in (2.10) satisfies $\Psi_{1} \in L^{2}(k, \infty)$ for every $k \in \mathbb{R}$ if and only if the following two conditions are fulfilled:

$$
\begin{gathered}
a+c>0, \\
\frac{b+c}{\sqrt{2 c}}+\sqrt{2(a+c)}+\frac{1}{2} \in \mathbb{N}_{0} .
\end{gathered}
$$


Note that the second condition forces the hypergeometric function $U$ in (2.10) to become a polynomial.

(ii) The solution $\Psi_{2}$ given in (2.11) satisfies $\Psi_{2} \in L^{2}(k, \infty)$ for every $k \in \mathbb{R}$ if and only if the following condition is fulfilled:

$$
a+c>0 .
$$

Here, it is not necessary that the hypergeometric function ${ }_{1} F_{1}$ in $(2.11)$ becomes a polynomial.

If the above conditions are satisfied, we have as a special case $\Psi \in L^{2}\left(\mathbb{R}^{+}\right)$. Note that we can never obtain $\Psi \in L^{2}(\mathbb{R})$ if $\Psi$ is nontrivial.

\subsection{Solution and $L^{2}-$ Normalizability for $c=0$}

In this case, the solutions in (2.10), (2.11) are not defined, and the following solutions can be used instead:

$$
\begin{aligned}
& \Psi_{1}(x)=J_{\sqrt{8 a}}\left(\sqrt{8 b\left(\tanh ^{2}(x)-1\right)}\right), \\
& \Psi_{2}(x)=J_{-\sqrt{8 a}}\left(\sqrt{8 b\left(\tanh ^{2}(x)-1\right)}\right),
\end{aligned}
$$

where $J$ is the Bessel function of the first kind [15]. If the order of the Bessel function is a natural number, that is, if there is a natural number $n$, such that $a$ can be written as

$$
a=\frac{1}{8} n^{2}
$$

then the following solution has to be used instead of (2.14) and (2.15):

$$
\begin{aligned}
& \Psi_{1}(x)=J_{n}\left(\sqrt{8 b\left(\tanh ^{2}(x)-1\right)}\right), \\
& \Psi_{2}(x)=Y_{n}\left(\sqrt{8 b\left(\tanh ^{2}(x)-1\right)}\right),
\end{aligned}
$$

where $Y$ is the Bessel function of the second kind [15]. Note that the first solutions (2.10), (2.11) can be written in a more compact way by means of Whittaker functions [15]. We do not introduce these functions here, as this would affect the transparency of subsequent calculations. The following conditions for $L^{2}$-normalizability apply. 
(i) The solution $\Psi_{1}$ given in (2.14) satisfies $\Psi_{1} \in L^{2}(\mathbb{R})$ if and only if the following condition is fulfilled:

$$
a>0 .
$$

(ii) The solution $\Psi_{1}$ given in (2.17) satisfies $\Psi_{1} \in L^{2}(\mathbb{R})$ if and only if the following condition is fulfilled:

$$
n \geq 1 \text {. }
$$

(iii) The solutions $\Psi_{2}$ given in (2.15) and (2.18) are not $L^{2}$-normalizable on unbounded intervals.

\section{Proof of Regularity Results}

Due to the continuity of the solutions given in the last section, we only have to check $L^{2}$ normalizability at positive and negative infinity. In order to simplify notation, let us set $z=$ $\tanh (x)$. Clearly, $z \rightarrow \pm 1$ if $x \rightarrow \pm \infty$.

\section{1. $L^{2}$-Normalizability of the Solution for $c \neq 0$}

We first study $L^{2}$-normalizability of solution (2.10) at positive infinity. To this end, we assume that the first argument of the confluent hypergeometric function $U$ is not a negative integer or zero:

$$
-\frac{b+c}{\sqrt{2 c}}+\sqrt{2(a+c)}+\frac{1}{2} \notin-\mathbb{N}_{0}
$$

If this condition is not fulfilled, then the confluent hypergeometric function becomes a polynomial in its third argument, which will be treated as a separate case below. We now take the function $U$ from (2.10) and expand it into the Taylor series around infinity, that is, around $z=1$ :

$$
\begin{gathered}
U\left(-\frac{b+c}{\sqrt{2 c}}+\sqrt{2(a+c)}+\frac{1}{2}, \sqrt{8(a+c)}+1, \sqrt{8 c}(1-z)\right) \\
=(1-z)^{-\sqrt{8(a+c)}}+O\left((1-z)^{1-\sqrt{8(a+c)}}\right) .
\end{gathered}
$$

Inspection of (2.10) shows that its behaviour at positive infinity $(z=1)$ is governed by the following expression:

$$
\begin{aligned}
\Psi_{1}(x) & =(1-\tanh (x))^{\sqrt{2(a+c)}-\sqrt{8(a+c)}} \\
& =(1-\tanh (x))^{-\sqrt{2(a+c)}} .
\end{aligned}
$$


Hence, if $a+c \geq 0$, then the differential probability amplitude $\left|\Psi_{1}\right|^{2} d x$ around infinity is given by (note that $d x=\left(1-z^{2}\right)^{-1} d z$ )

$$
\begin{aligned}
\left|\Psi_{1}(x)\right|^{2} d x & =\left|(1-\tanh (x))^{-2 \sqrt{2(a+c)}}\right| d x \\
& =\left|(1-z)^{-2 \sqrt{2(a+c)}} \frac{1}{1-z^{2}}\right| d z \\
& =\left|(1-z)^{-2 \sqrt{2(a+c)}-1} \frac{1}{1+z}\right| d z .
\end{aligned}
$$

Since the exponent of $1-z$ is always smaller than minus one, $\left|\Psi_{1}\right|^{2} d x$ is not integrable on any interval $(k, 1)$ for a real $k \geq 0$. If $a+c<0$, we have that

$$
\left|\Psi_{1}(x)\right|^{2} d x=\left|(1-z)^{-1} \frac{1}{1+z}\right| d z .
$$

Again, the exponent of $1-z$ is always minus one, implying that $\left|\Psi_{1}\right|^{2} d x$ is not integrable on any interval $(k, 1)$ for a real $k \geq 0$. Consequently, $\Psi_{1}$ is not $L^{2}$-normalizable around infinity. Next, let us consider the case where condition (3.1) is not satisfied; that is, we assume

$$
n:=-\frac{b+c}{\sqrt{2 c}}+\sqrt{2(a+c)}+\frac{1}{2} \in-\mathbb{N}_{0} .
$$

In this case, the confluent hypergeometric function $U$ becomes a polynomial of degree $n$ [15]. Hence, the solution $\Psi_{1}$ in (2.10) behaves at positive infinity $(z=1)$ as follows:

$$
\Psi_{1}(x)=(1-\tanh (x))^{\sqrt{2(a+c)}} .
$$

If $a+c \geq 0$, then the differential probability amplitude $\left|\Psi_{1}\right|^{2} d x$ around infinity is given by

$$
\begin{aligned}
\left|\Psi_{1}(x)\right|^{2} d x & =\left|(1-\tanh (x))^{2 \sqrt{2(a+c)}}\right| d x \\
& =\left|(1-z)^{2 \sqrt{2(a+c)}} \frac{1}{1-z^{2}}\right| d z \\
& =\left|(1-z)^{2 \sqrt{2(a+c)}-1} \frac{1}{1+z}\right| d z .
\end{aligned}
$$
only if

The latter expression remains integrable on each interval $(k, 1)$ for a real $k \geq 0$ if and

$$
a+c>0
$$

If $a+c<0$, then we have the same situation as in (3.5), implying that $\Psi_{1}$ is not $L^{2}$ normalizable around infinity. Let us now study the $L^{2}$-normalizability of the second solution 
(2.11) at infinity. Although the confluent hypergeometric function ${ }_{1} F_{1}$ becomes a polynomial if its first argument is a negative integer or zero, we do not need to treat the latter case separately, as we did for the function $U$. This is due to the fact that $\operatorname{around} \tanh (x)=1$ we always have

$$
{ }_{1} F_{1}\left(-\frac{b+c}{\sqrt{2 c}}+\sqrt{2(a+c)}+\frac{1}{2}, \sqrt{8(a+c)}+1, \sqrt{8 c}(1-\tanh (x))\right)=1+O(\tanh (x)-1) .
$$

Now, the inspection of solution (2.11) shows the following asymptotic behaviour at infinity:

$$
\Psi_{2}(x)=(1-\tanh (x))^{\sqrt{2(a+c)}}
$$

Thus, if $a+c \geq 0$, then we get for the differential probability amplitude $\left|\Psi_{2}\right|^{2} d x$ around infinity (recall that $z:=\tanh (x)$ )

$$
\begin{aligned}
\left|\Psi_{2}(x)\right|^{2} d x & =\left|(1-\tanh (x))^{2 \sqrt{2(a+c)}}\right| d x \\
& =\left|(1-z)^{2 \sqrt{2(a+c)}-1} \frac{1}{1+z}\right| d z .
\end{aligned}
$$

Thus, the latter expression remains integrable at infinity $(z=1)$ if and only if

$$
a+c>0
$$

As before, if $a+c<0$, then we have the same situation as in (3.5), implying that $\Psi_{1}$ is not $L^{2}$-normalizable on $\mathbb{R}$. Next, we study $L^{2}$-normalizability of solutions (2.10) and (2.11) at negative infinity. Both hypergeometric functions $U$ and ${ }_{1} F_{1}$ that appear in (2.10) and (2.11) are bounded at minus infinity, since their last argument turns there into the finite value $2 \sqrt{8 c}$. Thus, since solutions (2.10) and (2.11) differ only in the type of hypergeometric function, they present the same asymptotic behaviour at negative infinity. Inspection of (2.10) and (2.11) shows that both solutions take a finite value there. This gives with $z=\tanh (x)$

$$
\begin{aligned}
\left|\Psi_{1}(x)\right|^{2} d x & =\left|\frac{1}{1-z^{2}}\right| d z \\
& =\left|(1+z)^{-1} \frac{1}{1-z}\right| d z .
\end{aligned}
$$

The latter expression is not integrable at $z=-1$, and therefore solution (2.10) is not $L^{2}$ normalizable on any interval $(-\infty, k)$ for a real number $k$. Since we have seen that both solutions (2.10) and (2.11) show the same behaviour at negative infinity, solution (2.11) is not $L^{2}$-normalizable on any interval $(-\infty, k)$ for a real number $k$. 


\section{2. $L^{2}$-Normalizability of the Solution for $c=0$}

We now turn to the pair of solutions (2.14) and (2.15). First note that each of these solutions has the same asymptotic behaviour at positive and negative infinity, because the function tanh appears in its second power only. We have at infinity

$$
J_{ \pm \sqrt{8 a}}\left(\sqrt{8 b\left(\tanh ^{2}(x)-1\right)}\right)=(\tanh (x)-1)^{ \pm \sqrt{8 a}}+O\left((\tanh (x)-1)^{ \pm \sqrt{8 a}+1}\right)
$$

This gives the following differential probability amplitude around infinity (as before, corresponding to $z=1$ ) for $a \geq 0$ :

$$
\begin{aligned}
\left|\Psi_{1}(x)\right|^{2} d x & =\left|(z-1)^{\sqrt{8 a}} \frac{1}{1-z^{2}}\right| d z \\
& =\left|(z-1)^{\sqrt{8 a}-1} \frac{1}{1+z}\right| d z .
\end{aligned}
$$

The latter expression remains integrable at $z=1$ (and therefore at $z=-1$ ) if and only if

$$
a>0 .
$$

If $a<0$, we get

$$
\left|\Psi_{1}(x)\right|^{2} d x=\left|(z-1)^{-1} \frac{1}{1+z}\right| d z
$$

which is not integrable at $z=1$. We find for the second probability amplitude around infinity for $a \geq 0$ that

$$
\begin{aligned}
\left|\Psi_{2}(x)\right|^{2} d x & =\left|(z-1)^{-\sqrt{8 a}} \frac{1}{1-z^{2}}\right| d z \\
& =\left|(z-1)^{-\sqrt{8 a}-1} \frac{1}{1+z}\right| d z .
\end{aligned}
$$

This is not integrable at $z=1$. If $a<0$, the same situation as in (3.18) occurs. Let us finally derive $L^{2}$-normalizability conditions for the last pair of solutions (2.17) and (2.18). The asymptotic behaviour of these solutions is the same at positive and negative infinity. In addition, it is the same as in (3.15); only the argument of the Bessel function must be replaced by $n$ :

$$
\begin{aligned}
& J_{n}\left(\sqrt{8 b\left(\tanh ^{2}(x)-1\right)}\right)=(\tanh (x)-1)^{n}+O\left((\tanh (x)-1)^{n+1}\right), \\
& Y_{n}\left(\sqrt{8 b\left(\tanh ^{2}(x)-1\right)}\right)=(\tanh (x)-1)^{-n}+O\left((\tanh (x)-1)^{-n+1}\right) .
\end{aligned}
$$


Thus, the results obtained for the second solution set (2.14), (2.15) apply here. This means that the first solution (2.17) is $L^{2}$-normalizable if and only if

$$
n \geq 1
$$

whereas the second solution (2.18) is never $L^{2}$-normalizable.

\section{Concluding Remarks}

We have presented a careful analysis of the Schrödinger equation for a third-order hyperbolic potential and an effective mass step. Since the hyperbolic potential (2.3) and its special cases are useful models for interatomic and intermolecular forces, this paper motivates further studies in order to find more combinations of intermolecular potentials and physically meaningful effective mass functions that lead to closed-form solutions of the corresponding Schrödinger equation.

\section{References}

[1] C. Grosche, "Path integral solutions for deformed Pöschl-Teller-like and conditionally solvable potentials," Journal of Physics A, vol. 38, no. 13, pp. 2947-2958, 2005.

[2] H. Eğrifes, D. Demirhan, and F. Büyükkılıç, "Exact solutions of the Schrödinger equation for the deformed hyperbolic potential well and the deformed four-parameter exponential type potential," Physics Letters A, vol. 275, no. 4, pp. 229-237, 2000.

[3] H. Eğrifes, D. Demirhan, and F. Büyükkılıç, "Exact solutions of the Schrödinger equation for two deformed hyperbolic molecular potentials," Physica Scripta, vol. 60, no. 3, pp. 195-198, 1999.

[4] T. Gora and F. Williams, "Electronic states of homogeneous and inhomogeneous mixed semiconductors," in II-VI Semiconducting Compounds, D. G. Thomas, Ed., Benjamin, New York, NY, USA, 1967.

[5] T. Gora and F. Williams, "Theory of electronic states and transport in graded mixed semiconductors," Physical Review, vol. 177, no. 3, pp. 1179-1182, 1969.

[6] G. T. Landsberg, Solid State Theory: Methods and Applications, Wiley-Interscience, London, UK, 1969.

[7] A. D. Alhaidari, "Solutions of the nonrelativistic wave equation with position-dependent effective mass," Physical Review A, vol. 66, no. 4, Article ID 042116, 7 pages, 2002.

[8] B. Bagchi, P. S. Gorain, and C. Quesne, "Morse potential and its relationship with the Coulomb in a position-dependent mass background," Modern Physics Letters A, vol. 21, no. 36, pp. 2703-2708, 2006.

[9] B. Bagchi, P. Gorain, C. Quesne, and R. Roychoudhury, "Effective-mass Schrödinger equation and generation of solvable potentials," Czechoslovak Journal of Physics, vol. 54, no. 10, pp. 1019-1025, 2004.

[10] A. Ganguly and L. M. Nieto, "Shape-invariant quantum Hamiltonian with position-dependent effective mass through second-order supersymmetry," Journal of Physics A, vol. 40, no. 26, pp. 72657281, 2007.

[11] T. Tanaka, " $N$-fold supersymmetry in quantum systems with position-dependent mass," Journal of Physics A, vol. 39, no. 1, pp. 219-234, 2006.

[12] L. Dekar, L. Chetouani, and T. F. Hammann, "An exactly soluble Schrödinger equation with smooth position-dependent mass," Journal of Mathematical Physics, vol. 39, no. 5, pp. 2551-2563, 1998.

[13] L. Dekar, L. Chetouani, and T. F. Hammann, "Wave function for smooth potential and mass step," Physical Review A, vol. 59, no. 1, pp. 107-112, 1999.

[14] K. C. Yung and J. H. Yee, "Derivation of the modified Schrödinger equation for a particle with a spatially varying mass through path integrals," Physical Review A, vol. 50, no. 1, pp. 104-106, 1994.

[15] I. S. Gradshteyn and I. M. Ryzhik, Table of Integrals, Series, and Products, Academic Press, Boston, Mass, USA, 1994. 


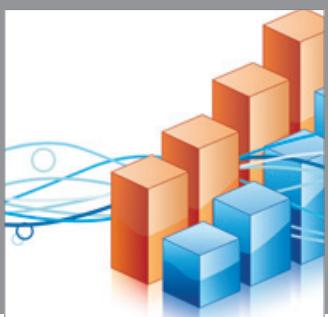

Advances in

Operations Research

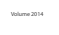

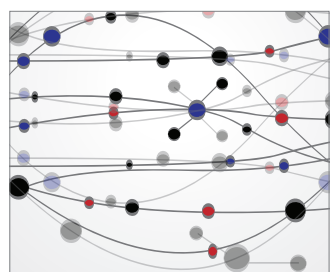

\section{The Scientific} World Journal
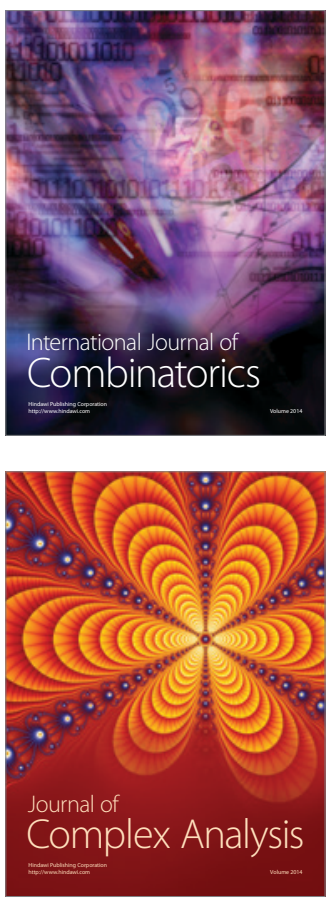

International Journal of

Mathematics and

Mathematical

Sciences
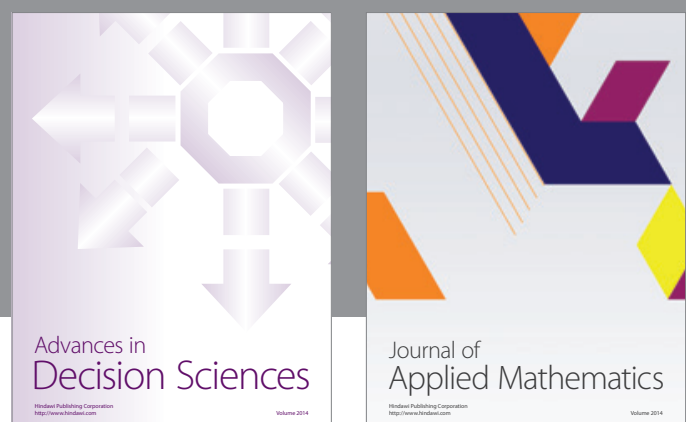

Journal of

Applied Mathematics
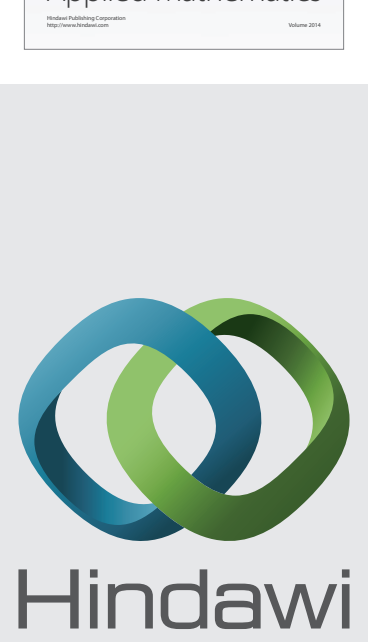

Submit your manuscripts at http://www.hindawi.com
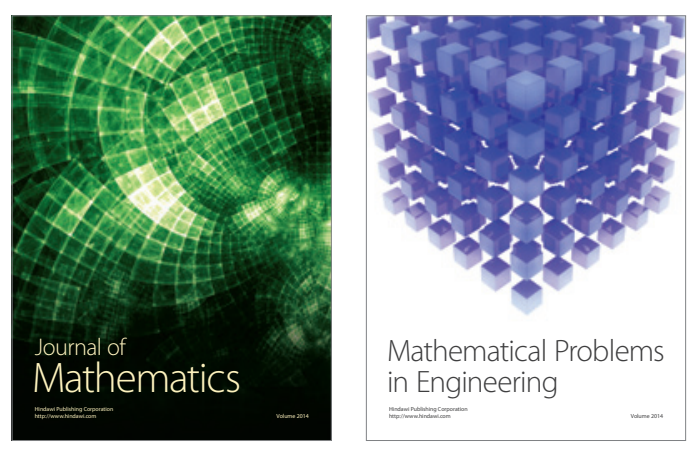

Mathematical Problems in Engineering
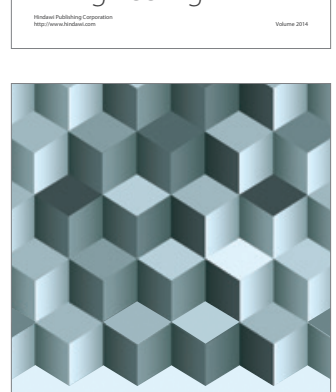

Journal of

Function Spaces
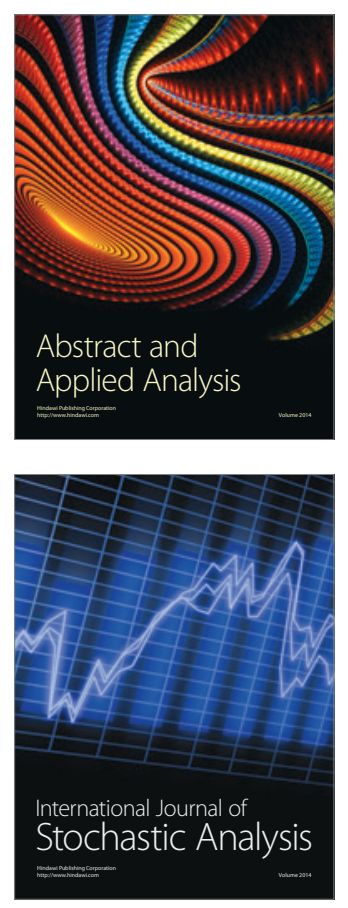

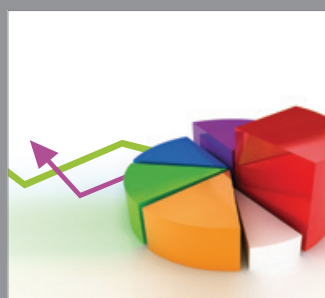

ournal of

Probability and Statistics

Promensencen
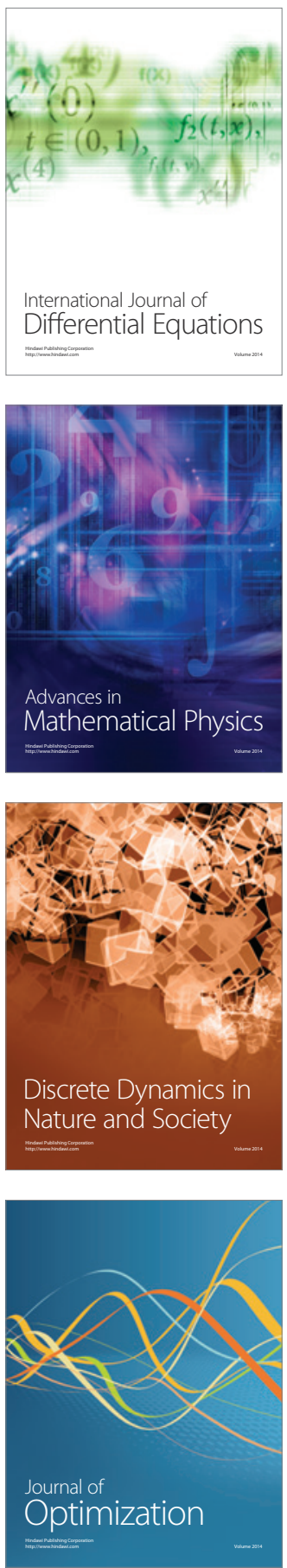